Physics

Weights \& Measures fields

\title{
A new position sensor for high-speed measurement of multiple points
}

\author{
Mitsuru Baba \\ Okayama University \\ Yuko Hori \\ Okayama Univeristy
}

\author{
Tadataka Konishi \\ Okayama University \\ Kouji Otani \\ Okayama University
}

This paper is posted at eScholarship@OUDIR : Okayama University Digital Information Repository.

http://escholarship.lib.okayama-u.ac.jp/weights_and_measures/1 
IEEE Instrumentation and Measurement Technology Conference

Brussels, Belgium, June 4-6, 1996

\title{
A New Position Sensor for High-Speed Measurement of Multiple Points
}

\author{
Mitsuru Baba, Tadataka Konishi, Yuko Hori and Kouji Otani \\ Faculty of Engineering Okayama University \\ Tsushima-naka 3-1-1, Okayama,Okayama, 700, Japan
}

Phone 81-86-252-1111 Fax 81-86-255-9136 Email baba@sdc.it.okayama-u.ac.jp

\begin{abstract}
A high-speed measuring system of multiple points is becoming an important issue in many industrial applications. Therefore, the development of a high-speed position sensor is an important issue. However, conventional sensors such as CCD(charge-coupled device) and PSD(position-sensitive detector) are insufficient to apply to the high-speed measurement of multiple points. We propose a new position sensor for high-speed measurement of multiple points. The proposed sensor features a single scanning detecting method of multiple points by parallel processing technique and design of the sensor by analog circuitry, which makes high-speed measurement of multiple points possible. The designed sensor system realizes both high-speed performance and high accuracy.
\end{abstract}

\section{INTRODUCTION}

A measuring system of multiple points is becoming an important issue in many industrial applications such as automatic inspection of manufactured parts and robot vision[1]. In these applications, short measuring time is often required along with high accuracy. Therefore, a development of a high-speed position sensor is an important issue[2].

Conventional sensors such as CCD(charge-coupled device)[3] and PSD(position-sensitive detector)[4] perform well as position sensors. However, they are insufficient to apply to the high-speed measurement of multiple points.

PSD is not a scanning type sensor. It is a high-speed position sensor. However, PSD cannot apply to simultaneous measurement of plural points[2]. Besides, PSD is subject to significant position measurement errors arising from background light[5]. Accordingly, a practical measuring system requires compensation for background light striking on PSD, and cannot utilize the high-speed performance of PSD.
The basic structure of CCD is that of a row of imaging elements and an analog shift register consisting of a series of closely spaced capacitors[3]. CCD can easily to measure multiple points and separate signal light from background light because the amount of charge collected at each imaging element is proportional to illumination intensity at its location[6]. However, CCD has difficulty in acquiring position data at high-speed due to scanning time of the analog shift register.

Therefore, a development of a new sensor based on a concept different from conventional sensors is necessary to greatly improve the measuring speed of multiple points. We propose a new position sensor for high-speed measurement of multiple points. This paper describes a principle of the proposed sensor, a circuitry of the sensor system and experimental results by the test sensor system mounted using the discrete IC devices.

\section{II . METHOD OF POSITION MEASUREMENT}

\section{A. Sensor Structure}

There are two structural types of the proposed sensor available, a line type and an area type. The sensor uses much fewer sensor elements than the other array sensors such as CCD, and detects multiple spotlike signals such as spot lights. In this paper, we explain only the line type. The area type will not be presented here because a principle of the area type is similar to that of the line type. The sensor consists of a small number of sensor elements for a more compact sensor system, and it interpolates between measured data for high accuracy measurement. 


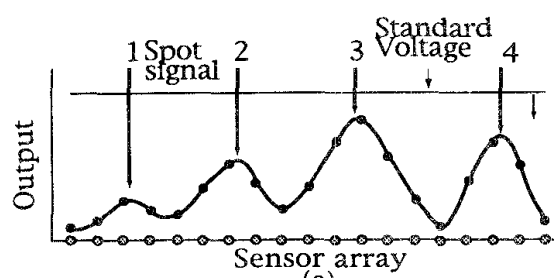

(a)

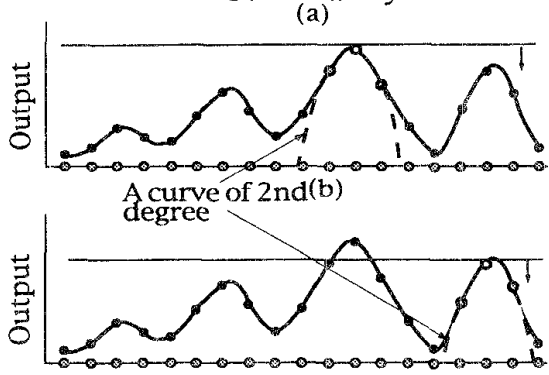

(c)

Fig.1 Measuring process

\section{B. Measuring Process}

Fig. 1 shows the processes of the position measurement in this sensor. This sensor uses parallel processing technique, and the process of the operation is as follows. (1)In order to detect plural sensor elements outputting peak value of sensor array, the sensor sets a standard voltage as shown in Fig.1(a). In this figure, an example that four spot signals are inputted to the sensor is showed. First, the sensor initializes the standard voltage to a larger value than maximum values of all sensor elements.

(2)Secondly, the sensor lowers the standard voltage and detects the peak value of the sensor array by comparing it with the standard voltage. Actually, a true peak position exists between sensor elements. Therefore, as soon as the standard voltage coincides with the peak value, the sensor interpolates by a curve of second degree between peak values and values of two sensor elements adjacent to sensor element outputting peak value, and determines the true peak as shown in Fig.1(b). (3)Subsequently, the sensor lowers the voltage to detect the next peak value of the sensor array without stopping descent of the voltage.

(4)When the sensor detects the next peak value as shown in Fig.1(c), it interpolates between the above-mentioned values of three sensor elements as lowered the voltage. (5)These processes are repeated until the standard voltage reaches zero. Consequently, the sensor can detect all peak values.

An advantage of the sensor is the parallel processing technique, which carries out both the lowering of the standard voltage and the calculating of true positions simultaneously.

\section{Limitations of the Sensor}

The sensor has the following limitations

(1)The relationship between input and output of sensor element must have linear characteristics.

(2)Signals inputting to sensor elements need to be spotlike signals.

(3)Each peak value must differ from each other.

(4)A distance between two sensor elements outputting peak value must be separated by four elements at least. Therefore, the maximum number of peaks that the sensor can detect equals a quarter of the total numbers of sensor elements.

If the sensor elements satisfy the above-mentioned conditions, available sensor elements include photodiode, ultrasonic sensor and pressure sensor.

\section{III . SENSOR SYSTEM CONFIGURATION}

\section{A. An Outline of Sensor System}

Fig. 2 shows a block diagram of the proposed sensor system mounted using analog circuits mainly. Essential components in this sensor system include a sensor array unit, a sensor element signal processing unit, a peak hold unit, a standard voltage generating unit, a peak detecting unit, a position signal processing unit, an output selecting unit, an analog operating unit and an output sample/hold unit.

When mulciple spot signals are inputted to sensor array, the sensor element signal processing unit amplifies each signal of sensor elements. The peak hold unit holds these amplified signals and transmits the signal to the detecting unit.

The standard voltage generating unit generates the voltage which lowers at constant speed, and then the voltage is transmitted to the peak detecting unit.

The peak detecting unit compares each held value of sensor elements with the standard voltage, and then generates a position information of sensor elements that coincide with the standard voltage.

The position signal processing unit selects two sensor 


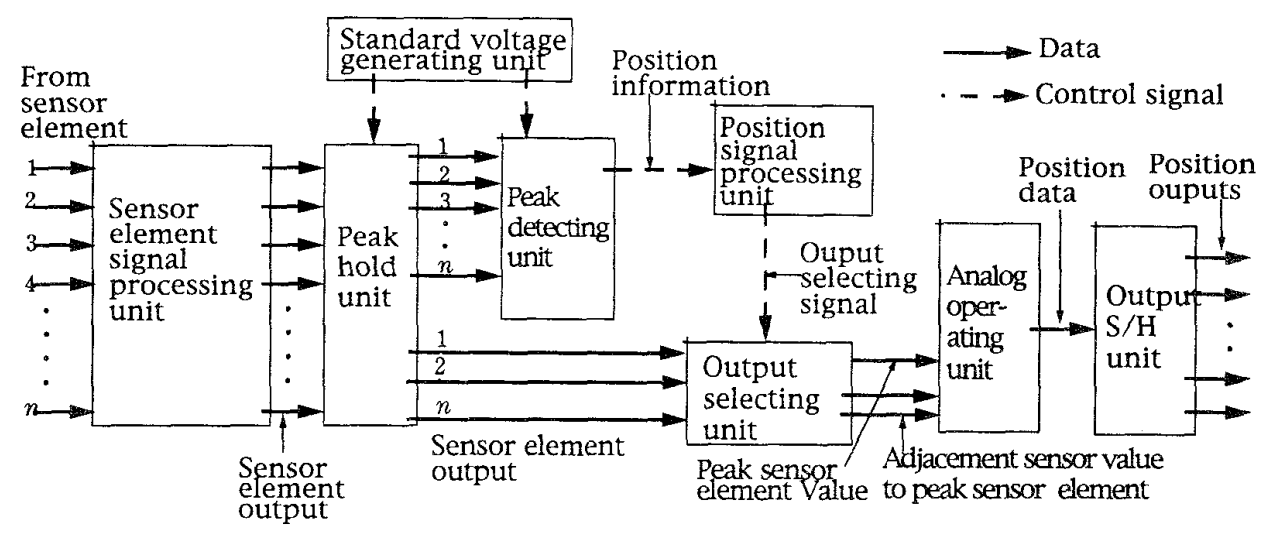

Fig. 2 Block diagram of sensor system

elements adjacent to sensor element of peak position on both sides by use of position information transmitted from the peak detecting unit. This generates an output selecting signal and transmits the selecting signal to the output selecting unit.

The output selecting unit samples the outputs of two sensor elements adjacent to sensor element outputting peak value on both sides by this selecting signal. The analog operating unit calculates a true spot position by interpolating between three values, and then transmits the calculating values to the output sample/hold unit. The output sample/hold unit holds the calculating values and outputs the values as position outputs. While these process are carried out, the sensor continues to lower the standard voltage in order to detect the next sensor elements outputting peak value. When the voltage reaches zero, the whole true spot position are determined.

We designed the sensor system by analog circuit mainly, which enables the measurement of multiple positions at high-speed.

\section{B. Circuities of the Sensor System}

We present here novel parts of circuities of the sensor system .

Fig. 3 illustrates a circuitry of the peak detecting unit. The important consideration in this circuitry is a method for both detecting sensor elements of outputting peak value and holding the information of the position. We successfully achieved the method with a comparator, 3-input NAND gate and 2-input OR gate corresponding to sensor element.

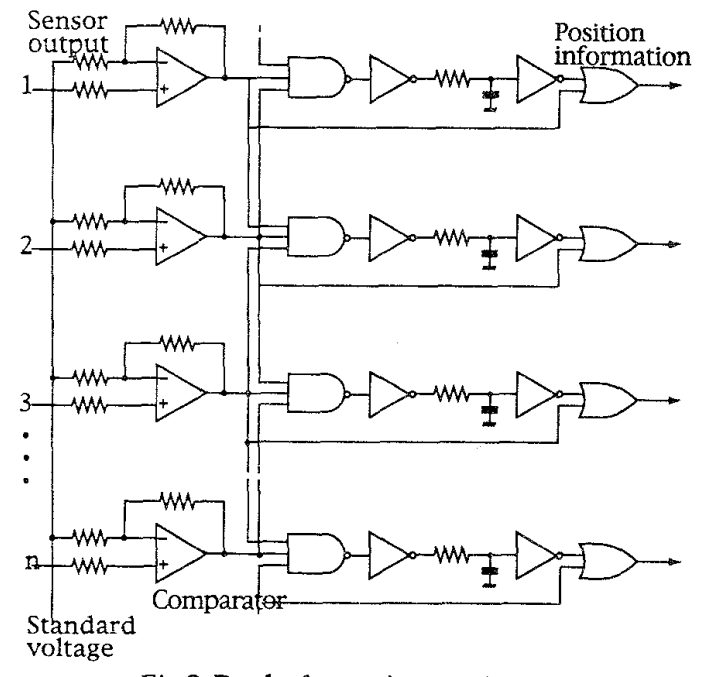

Fig3 Peak detecting unit

Fig. 4 shows a timing diagram of the signal of the comparator, the 3-input NAND gate and the 2-input OR gate as shown in Fig.3. The operation is as follows. First, the initial outputs of comparator are set at H-level. When the lowering standard voltage coincides with a value of the sensor element outputting maximum peak value, the comparator output corresponding to the sensor element is changed from $\mathrm{H}$-level to L-level. As the standard voltage lowers, the comparator outputs are changed from $\mathrm{H}$-level to L-level in order of decreasing sensor element output. Next, three neighboring comparator outputs are inputted to 3-input NAND gate and are delayed by delay circuit as shown in Fig.4. Then both this delayed output and the comparator output are inputted to 2-input OR gate simultaneously. As a 

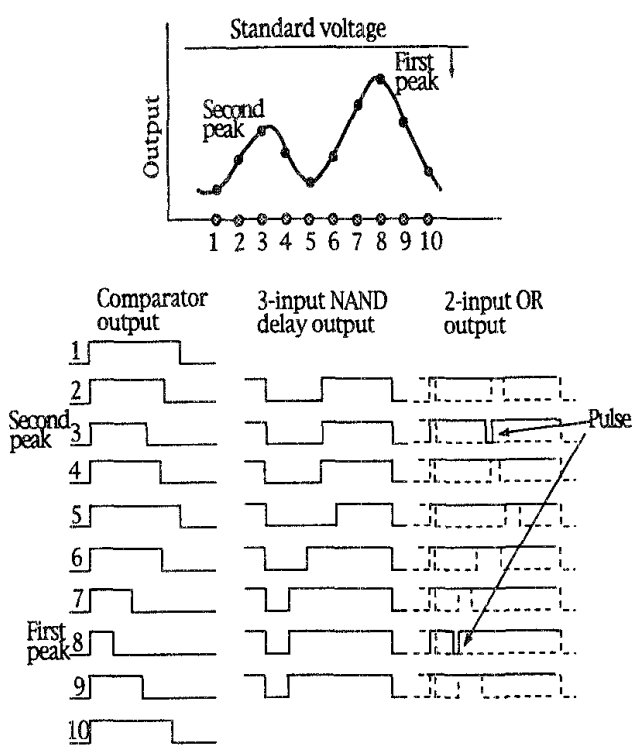

Fig4 Timing diagram of the peak detecting unit

result, only OR gate output corresponding to peak position sensor element generates a narrow negative pulse. This negative pulse is defined as position information.

Fig. 5 illustrates a circuitry of the position signal processing unit. The position information generated by peak detecting unit sets the flip-flop, and then Q output of flip-flop is inputted to 8-to-3 line priority encoder. The encoder generates an output selecting signal for identifying first peak position sensor element as four bit signal. Next, the output selecting signal is inputted to both output selecting unit and 4-to-16 line decoder.

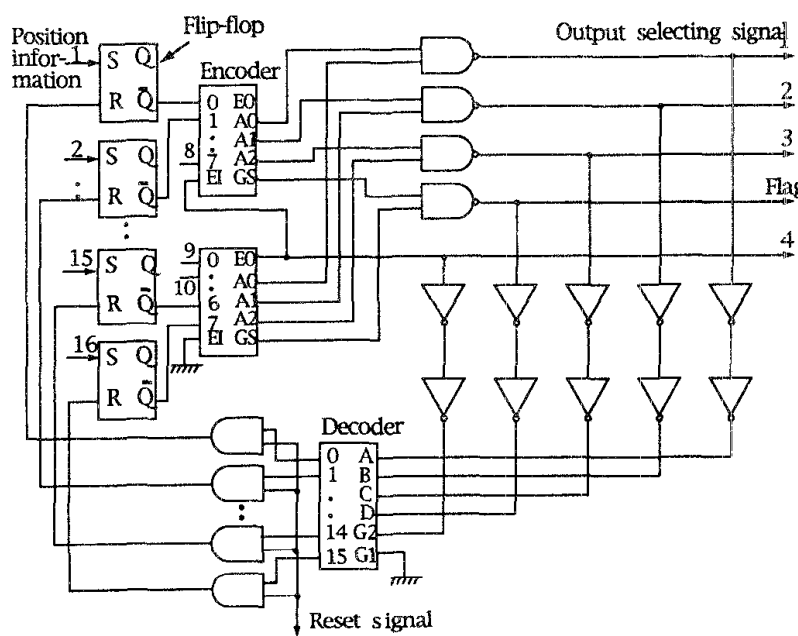

Fig.5 Position signal processing unit
The decoder resets the flip-flop set in above-mentioned process. This enables the second peak position sensor element to generate a second output selecting signal. Repeation of these processes enables us to measure the position of multiple points.

Fig. 6 shows a circuitry of the output selecting unit. As shown in Fig.6, by applying the output selecting signal to four multiplexer, we obtain the following four values; an output vi of peak position sensor element and its position $x i$; outputs $v i-1$ and $v i+1$ of two sensor elements adjacent to peak position sensor element on both sides. We obtain $v i-1$ and $v i+1$ by shifting the connection of the output line of sensor element and the input line of the multiplexer by one line before and behind. The position voltages corresponding to each sensor element generate by dividing a constant voltage.

Fig. 7 shows a circuitry of the analog operating unit. The four values of $x i, v i, v i-1$ and $v i+1$ are inputted to both operational amplifier and multiplexer. Thus, we can calculate the peak positions by the analog operating.

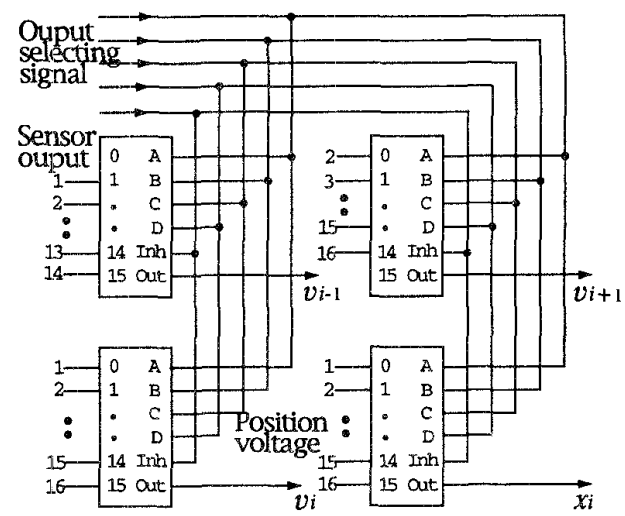

Fig.6 Output selecting unit

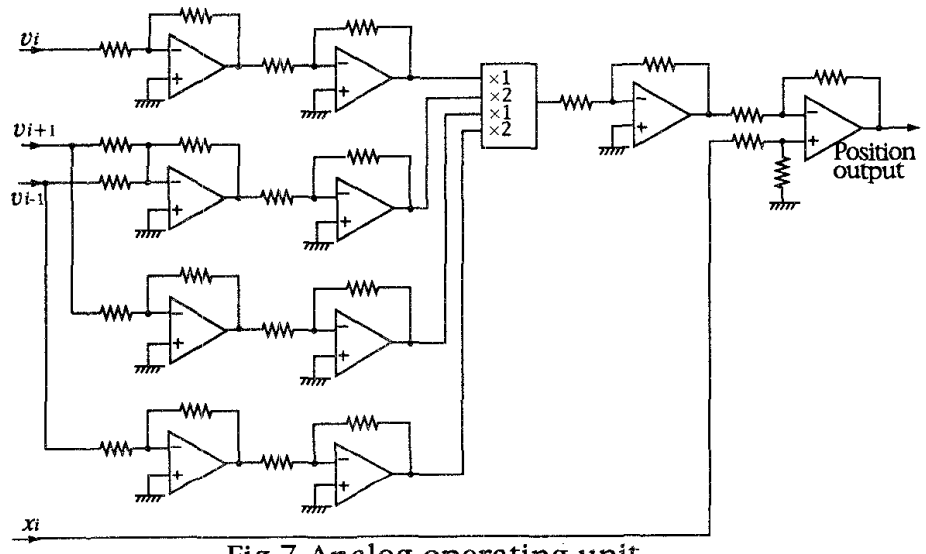

Fig.7 Analog operating unit 


\section{IV . EXPERIMENT}

\section{A. Test Circuitry}

We designed and constructed a test sensor circuit by use of discrete IC devices. The sensor array unit uses photodiode composed of 16 elements. We do an experiment with simultaneous position measurement for four points in order to confirm the proposed measuring principle.

\section{B. Experimental Results}

1) Accuracy: The procedure of experiment first sets sensor array unit and four light sources with semiconductor diode laser(emission wavelength $670 \mathrm{~nm}$ and output power $5 \mathrm{mw}$ ) to precision translation stages, and moves light sources as kept the distance.

The result in Fig. 8 shows the output signal versus the actual position. In this figure, four solid lines are drawn by the method of least squares. The measured data better matches solid lines. The deviations of measured data from linearity are within about 3.3\%.

2) Response: We do an experiment on measuring speed by using four light emitting diodes(LED). The result shows that this sensor can measure spot position signal at sampling time of $25 \mu \mathrm{s}$. This response is equivalent to $50 \mathrm{kHz}$ of measuring frequency and is proven to be highspeed compared to a conventional sensor.

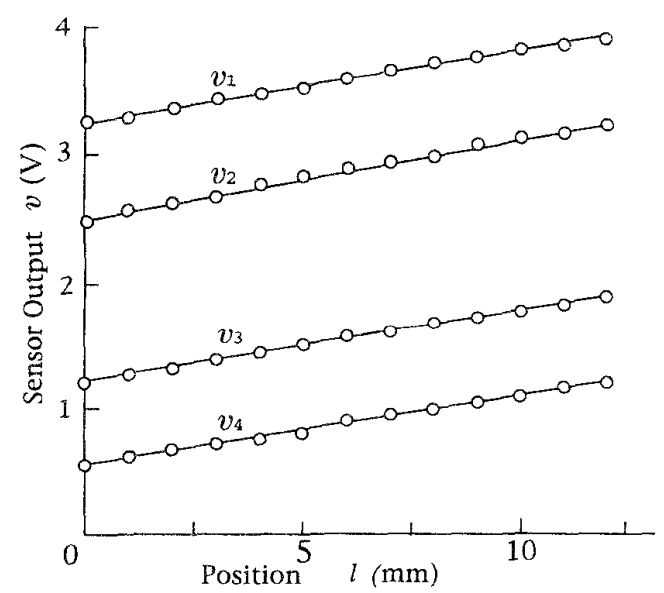

Fig8 Sensor output versus signal position

\section{$\checkmark$.DISCUSSION}

\section{A. Limitations of Accuracy}

The position measuring accuracy of the proposed sensor is determined by the difference between the following two ideal conditions and actual conditions.

(1) The distance between each neighboring sensor element must be the same.

(2)Any sensor element must output the same value when the same value is inputted.

We examined the position measuring errors arising from these differences theoretically. The computational results verify that these cause comparable errors.

\section{B. Comparison of the proposed sensor with both $C C D$} and $P S D$

The proposed sensor is such array-type as CCD on its structure on the one hand, and is such analog processing type as PSD on its signal processing on the other hand. As a result, the advantages of the proposed sensor over other position sensor such as CCD and PSD are as follows. (1)The proposed sensor can measure positions of multiple points by single scanning in spite of array type. Therefore, the output data rate of the sensor is much faster than $\mathrm{CCD}$ which requires the same number of scanning as the number of pixels. The number of pixels in standard commercial CCD ranges from 128 to 6000. In addition, the measuring speed of the sensor is much faster than that of PSD because PSD is necessary to process sequentially for measurement of multiple points. (2)As the proposed sensor uses only three values close to spot signal for the calculating of the positions, the position data is insensitive to background light positioned away from spot signal. In contrast to this, the position data of PSD is sensitive to background light because PSD calculates the position by use of light intensity exposed to the whole area.

(3)As the proposed sensor is array type, it can replace the sensor elements with ones corresponding to the subject of measurement. We can apply the sensor to photodiode, ultrasonic sensor, pressure sensor ,etc. On the other hand, PSD is difficult to replace the sensor elements.

(4)The proposed sensor is easy to mount by standard electronics technology and, moreover, to package as IC owing to many reacting of simple circuitry. 
This sensor has the following technological problems that other sensors do not have

(1)The proposed sensor is sensitive to spotlike noise added to any value of three values that are used in calculating the position.

(2)The proposed sensor needs to adjust many parts of the circuitry in order to reduce errors resulting from two followings errors:(a)errors on mounting, and (b)errors by characteristic difference between each sensor element. However, there is not an appropriate adjusting method yet, and so far a trial-and error method is used in this research.

\section{Feasibility to use digital signal processing techniques}

One of the most important features in this sensor is to replace digital processing with analog electric circuit, which results in high-speed measurement. To make high accuracy measurement possible, the proposed sensor requires that the circuit devices with the same electronical characteristics are selected and each circuit constants are adjusted appropriately. However, in many cases, it is difficult for the proposed sensor to meet these requirements thoroughly.

On the other hand, a digital processing can easily to measure position accurately because it can deal with these requirements appropriately. From only an accurate viewpoint, it should replace analog circuits with digital processing. However, measuring speed will be much slower than that of the proposed sensor because the digital processing is executed sequentially.

\section{Typical application for this sensor}

The proposed sensor can be applied to pattern measurement and high-speed image sensor for three dimensional shape measurement. In pattern measurement, the sensor can be utilized to input the plural edge detecting points for shape recognition. In three dimensional shape measurement, it can be used to receive multi-slit ray at high-speed and simultaneously. Currently we are working on the abovementioned measurement by use of this sensor, and good results are obtained.

\section{CONCLUSIONS}

We propose a new position sensor for high-speed measurement of multiple points. The proposed sensor features a single scanning detecting method of multiple points by parallel processing technique and design of the sensor by analog circuitry, which makes high-speed measurement of multiple points possible. In addition, the sensor measured the true spot positions by interpolation of a curve of second degree with analog operating. Therefore, the accurate measurement is compatible with high-speed measurement. We designed and constructed a test sensor circuits with discrete IC devices.

Performance of the sensor can be summarized as follows: (1)detection of simultaneous multiple points.

(2)rapid response of sampling period $50 \mathrm{kHz}$.

(3)linearity of about $3.3 \%$.

\section{REFERENCES}

[1] Jorge L.C.Sanz(Ed), Advances in Macine Vision, New York, Springer-Verlag, 1989.

[2] D.Qian,W.Wang,I.J.Busch-Vishniac and A.B.Buckman,"A method for measurement of multiple light spot positions on one position-sensitive detector(PSD)",IEEE Trans IM,vol.42,pp14-18, February,1993.

[3] W.Gopel,J.Hesse and J.N.Zemel,Sensors volume 6 Optical Sensors,New York, VCH,1992.

[4] K.Yamamoto,S.Yamaguti and Terada,"New structure of twodimensional position sensitivesemiconductor detector and application," IEEE Trans.Ncul.Sci.,vol.32,pp438-442,1985.

[5]T.Aida, S.Serikawa, W.Nakajima,A.Yamasiata and E.Nagamura,"Detection of three dimensional coodinates by PSD camera with disturbance correction of background light",Trans. IEE Japan,vol.107-C,July,1987.

[6]A.Rosenfeld and A.C.Kak,Digital Image Processing,Academic Press, 1977. 